\title{
An Ecological Comparison of Floristic Composition in Seasonal Semideciduous Forest in Southeast Brazil: Implications for Conservation
}

\author{
Sérgio de Faria Lopes, Ivan Schiavini, Ana Paula Oliveira, and Vagner Santiago Vale \\ Post-Graduation in Ecology and Conservation of Natural Resources, Federal University of Uberlandia, P. O. Box 593, \\ 38400-902 Uberlandia, MG, Brazil \\ Correspondence should be addressed to Sérgio de Faria Lopes, defarialopes@gmail.com
}

Received 30 March 2011; Revised 6 May 2011; Accepted 9 May 2011

Academic Editor: Frank Gilliam

Copyright ( $) 2012$ Sérgio de Faria Lopes et al. This is an open access article distributed under the Creative Commons Attribution License, which permits unrestricted use, distribution, and reproduction in any medium, provided the original work is properly cited.

We examined floristic patterns of ten seasonal semideciduous forest sites in southeastern Brazil and conducted a central sampling of one hectare for each site, where we took samples and identified all individual living trees with $\mathrm{DBH}$ (diameter at breast height, $1.30 \mathrm{~m}) \geq 4.8 \mathrm{~cm}$. Arboreal flora totaled 242 species, 163 genera, and 58 families. Fabaceae (38 species) and Myrtaceae (20 species) were families with the largest number of species. Only Copaifera langsdorffii and Hymenaea courbaril occurred at all sites. Multivariate analysis (detrended correspondence analysis and cluster analysis) using two-way indicator species analysis (TWINSPAN) indicated the formation of a group containing seven fragments in which Siparuna guianensis was the indicator species. This analysis revealed that similarities between studied fragments were due mainly to the successional stage of the community.

\section{Introduction}

The extent of seasonal semideciduous forests (SSFs) in Brazil is underestimated because of its naturally fragmented distribution $[1,2]$. Semideciduous seasonal forests occur along the contact zone between Atlantic forest and the diagonal of opened formations [3-5], comprising three different scenarios: (1) in northeastern Brazil, the SSFs form a marked belt $(<50 \mathrm{~km})$ in transition between the coastal rainforest and semiarid formations (Caatinga), but also occur in enclaves of montane forests, the altitude marsh $[6,7] ;(2)$ the transition between the Cerrado and the coastal Atlantic forest in southeastern Brazil involves an extensive occurrence of SSFs to the south, up to the east of Paraguay and northeast of Argentina, forming a complex mosaic with the Cerrado vegetation in the west; (3) in southeastern Brazil, a large Araucaria forest confronts subtropical coastal Atlantic forest, and the SSF appear to the west and south as a transition to the Chaco forests, and to the southeast with fields or the southern pampas $[8,9]$, and beyond disjunct areas located in Mato Grosso and Tocantins states [10].
In southeast Brazil, SSFs are distributed widely in sites with a seasonal rainfall regime, characteristic of the Atlantic forest and Cerrado domains. In the Atlantic forest domain, SSF is the predominant typology, and in the Cerrado domain, SSF occurs in enclaves, associated with permanent or intermittent watercourses [12] and should be regarded as lato sensu Atlantic forest, since it presents a floristicstructural identity similar to forests of the Atlantic forest domain [12].

Semideciduous seasonal forests suffered the same degradation process as other Brazilian ecosystems. Since the 1970s, there was an accelerated replacement of natural vegetal formations to pasture and use for agriculture, transforming extensive areas into an important agriculture area for grain and fruit production and livestock [13]. This rural reorganization was determined by the II National Development Plan (NDP), which collaborated for a modern agricultural deployment. With increasingly intense adoption of mechanization and land use, SSFs were drastically reduced, with only a few remaining in Southeast Brazil. 
TABLE 1: Information of ten seasonal semideciduous forest fragments in southeastern Brazil analyzed regarding regional tree flora composition.

\begin{tabular}{lccccc}
\hline Site & Counties & Area (ha) & Latitude (S) & Longitude (W) & Altitude (m.a.s.l.) \\
\hline 1 & Araguari & 200 & $18^{\circ} 29^{\prime} 50^{\prime \prime}$ & $48^{\circ} 23^{\prime} 03^{\prime \prime}$ & 680 \\
2 & Ipiaçu & 40 & $18^{\circ} 43^{\prime} 39^{\prime \prime}$ & $49^{\circ} 56^{\prime} 22^{\prime \prime}$ & 530 \\
3 & Monte Carmelo & 119 & $18^{\circ} 44^{\prime} 59^{\prime \prime}$ & $47^{\circ} 30^{\prime} 56^{\prime \prime}$ & 910 \\
4 & Uberaba & 70 & $19^{\circ} 40^{\prime} 35^{\prime \prime}$ & $48^{\circ} 02^{\prime} 12^{\prime \prime}$ & 790 \\
5 & Uberlândia & 17,5 & $18^{\circ} 40^{\prime} 26^{\prime \prime}$ & $48^{\circ} 24^{\prime} 32^{\prime \prime}$ & 600 \\
6 & Uberlândia & 30 & $18^{\circ} 57^{\prime} 03^{\prime \prime}$ & $48^{\circ} 12^{\prime} 22^{\prime \prime}$ & 880 \\
7 & Uberlândia & 22,3 & $19^{\circ} 08^{\prime} 39^{\prime \prime}$ & $48^{\circ} 08^{\prime} 46^{\prime \prime}$ & 430 \\
8 & Uberlândia & 16 & $19^{\circ} 10^{\prime} 04^{\prime \prime}$ & $48^{\circ} 23^{\prime} 41^{\prime \prime}$ & 800 \\
9 & Uberlândia & 35 & $18^{\circ} 55^{\prime} 40^{\prime \prime}$ & $48^{\circ} 03^{\prime} 51^{\prime \prime}$ & 890 \\
10 & Uberlândia & $18^{\circ} 51^{\prime} 35^{\prime \prime}$ & $48^{\circ} 13^{\prime} 53^{\prime \prime}$ \\
\hline
\end{tabular}

TABle 2: Number of families and species, Shannon diversity index $\left(H^{\prime}\right)$, and Pielou evenness index $\left(J^{\prime}\right)$ from ten sites of seasonal semideciduous forest in southeastern Brazil.

\begin{tabular}{lcccc}
\hline Site & Families & Species & $H^{\prime}$ & $J^{\prime}$ \\
\hline 1 & 32 & 78 & 3,442 & 0,79 \\
2 & 38 & 98 & 3,97 & 0,866 \\
3 & 27 & 50 & 2,924 & 0,747 \\
4 & 34 & 88 & 3,275 & 0,733 \\
5 & 33 & 79 & 3,366 & 0,768 \\
6 & 37 & 86 & 3,705 & 0,832 \\
7 & 37 & 73 & 3,471 & 0,809 \\
8 & 36 & 98 & 3,778 & 0,824 \\
9 & 38 & 103 & 3,868 & 0,836 \\
10 & 41 & 88 & 3,509 & 0,784 \\
\hline Total & 58 & 242 & 4,616 & 0,840 \\
\hline
\end{tabular}

Habitat fragmentation transforms the original landscape into different dynamic units that continually modify its structure [14]. Furthermore, the occurrence of disturbance histories on different scales and heterogeneity environmental landscapes can influence the species composition of forest fragments [15] and form species richness patterns according to the fragment successional stage $[16,17]$.

Therefore, owing to the similar process of disturbance in southeastern Brazilian forest formations and being located in the same catchment area, it is expected that the remaining SSF will have the same floristic pattern.

This work aims to analyze the floristic composition and regional richness in the arboreal species of ten fragments of SSF in southeastern Brazil, in order to answer the following questions: (1) what is the alpha diversity in sampled fragments? (2) is there a floristic pattern that represents the SSF in the studied region? (3) is the beta diversity in SSF similar to patterns found in other tropical forests?

\section{Material and Methods}

2.1. Floristic, Geographic, and Climate Data. We elaborate a floristic list from the tree species compilation in ten semideciduous seasonal forest fragments (according to Veloso classification [18]), distributed in five counties of southeastern
Brazil (Table 1). Studied sites are located in the extreme west of Minas Gerais state, defined by geographic coordinates $18^{\circ}$ $29^{\prime}-19^{\circ} 40^{\prime} \mathrm{S}$ and $47^{\circ} 30^{\prime}-49^{\circ} 53^{\prime} \mathrm{W}$ (Figure 1 ).

The studied region is part of a relief global set, named the "Domain of Central Brazil Tropical Plateaus" by Ab'Saber [19], and the "Plateaus and elevated plains of the Paraná sedimentary basin" by the RADAM project [20]. The soils in the studied areas are predominantly red nonferric latossols (LV) [21]. The predominant climate in the studied region is tropical savanna (Aw Megathermic), characterized, according to Köppen classification [22], by rainy summers and dry winters. The clearly seasonal climate has two well-defined seasons, where the winter season (April to September) has approximately six months of drought, and the summer season (October to March) is warm and rainy. The average annual temperature lies between $23^{\circ} \mathrm{C}$ and $25^{\circ} \mathrm{C}$, with July the month with the lowest average temperature $\left(16^{\circ} \mathrm{C}\right)$. The annual rainfall ranges from 1160 to $1460 \mathrm{~mm}$ [23].

2.2. Data Collection and Analysis. We conducted a central sampling of one hectare from each fragment of SSF, in an attempt to exclude edge effect and to obtain uniform samples, avoiding the ecotones of the adjacent formations. Central sampling gave priority to sites without the influence of watercourses (gallery forest), savanna formations (cerrado sensu stricto), and others forestry formations (dry seasonal forest and cerradão), since these vegetation contact areas vary considerably between fragments and would lead to changes in the species listing of each area. In each central sampling, all individual living trees with a DBH (diameter at breast height, $1.30 \mathrm{~m}$ ) of $\geq 4.8 \mathrm{~cm}$ were recorded and identified.

We identified the species using literature, queries in herbarium, and specialists. For specific binomial formation, we employed the w3 Tropicos database [24]. We deposited fertile material to the Uberlandia Federal University herbaria (Herbarium Uberlandensis: HUFU). Vegetative samples of all species were deposited at the Laboratory of Plant Ecology at the same university. Species were classified into families, according to the Angiosperm Phylogeny Group III system [25].

We compiled all species present in the inventories to undertake the floristic composition analysis. We carried 


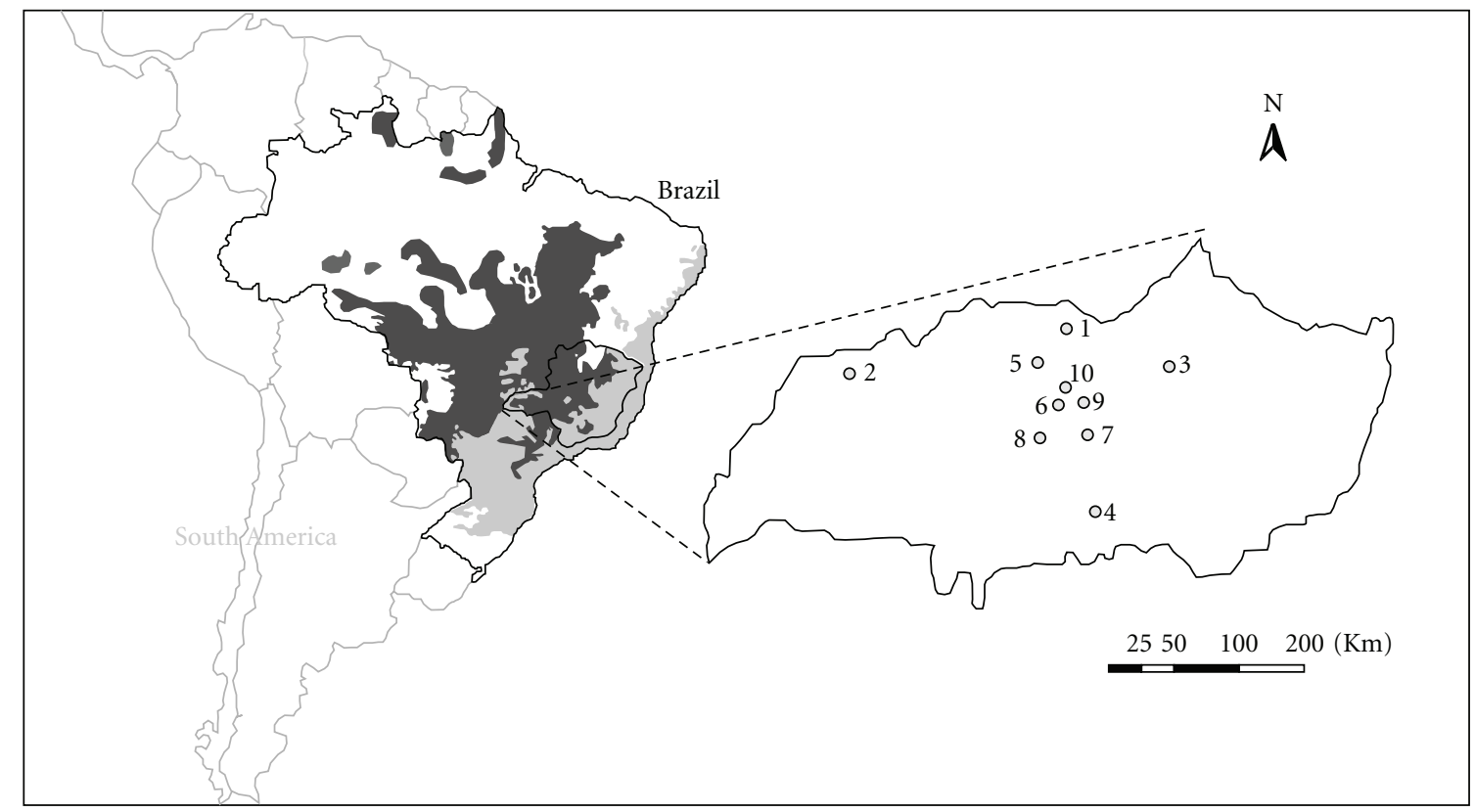

Cerrado domain

Atlantic forest domain

Figure 1: Location of the ten sites of seasonal semideciduous forest studied in the region of Triângulo Mineiro, Minas Gerais state, southeastern Brazil.

out the analysis by preparing a binary database (presence/absence), containing the tree species listed in each fragment. We only considered individual trees identified at the species level in this database composition. For the Alpha diversity evaluation, we used the Shannon diversity index $\left(H^{\prime}\right)$ and the Pielou evenness index $\left(J^{\prime}\right)$.

We used the binary database for the measurement of richness analysis, and also for the total number of species projection, we used first- and second-order, nonparametric jackknife estimators, in addition to Chao and Chao 2 estimators, which were capable of projecting total species richness from the samples of species richness [26], with 5,000 randomizations. We conducted the analysis using the EstimateS 8.0 program [27].

2.3. Multivariate Analyses. We did similarity and sorting analysis utilizing the FITOPAC SHELL 1.6.4 program [28], using an absolute density matrix for the species of ten sites, considering only those species with two or more occurrences, since species that had just one occurrence did not contribute to the floristic site ordering for floristic similarity. For categorical floristic data (presence and absence), we analyzed the similarity between sites, using the same absolute density matrix, transformed into presence/absence. We used the Sørensen similarity coefficient [29] and the unweighted pair group method with arithmetic mean (UPGMA) for a dendrogram graphical representation.

We applied multivariate analysis to quantitative tree species floristic data (abundance) present in all the compared fragments. For this, we realized a data ordination by using the detrended correspondence analysis (DCA, [30]). In addition, to define predominantly the indicator and preferred species of the floristic groups, based on frequency and density (species with $n \geq 5$ individuals), we used dichotomous hierarchical division using TWINSPAN (two-way indicator species analysis, [31]), from an absolute data matrix and its frequency at the 10 sites, with a cut level of $0,2,5$, and 10. These analyses were made by $\mathrm{PC}$-ord for Windows program version 4.0 [32].

\section{Results}

A total of 242 species, distributed in 165 genera and 58 families, were found in the SSF (Table 2). Families with a higher species richness were Fabaceae (sensu lato) (38 species), subdivided into Fabaceae Faboideae (20), Fabaceae Mimosoideae (11), Fabaceae Caesalpinioideae (5) and Fabaceae Cercideae (2), Myrtaceae (20 species), Rubiaceae (13), Annonaceae (11), Moraceae (10), Lauraceae (9), Meliaceae (9), and Malvaceae (8). These eight families showed a great contribution to the regional tree diversity, covering $48.8 \%$ of the species. Twenty-six families were represented by only one species. Annonaceae, Apocynaceae, Combretaceae, Fabaceae, Mimosoideae, Fabaceae, Faboideae, Lauraceae, Myrtaceae, Meliaceae, Rubiaceae, Salicaceae, Sapindaceae, and Sapotaceae were found at all ten studied sites.

Most well-represented genera are in Fabaceae Faboideae (13), Rubiaceae (13), Myrtaceae (10), Annonaceae (8), and Euphorbiaceae, Fabaceae Mimosoideae, and Malvaceae, with seven genera each. Approximately $30 \%$ of families were 


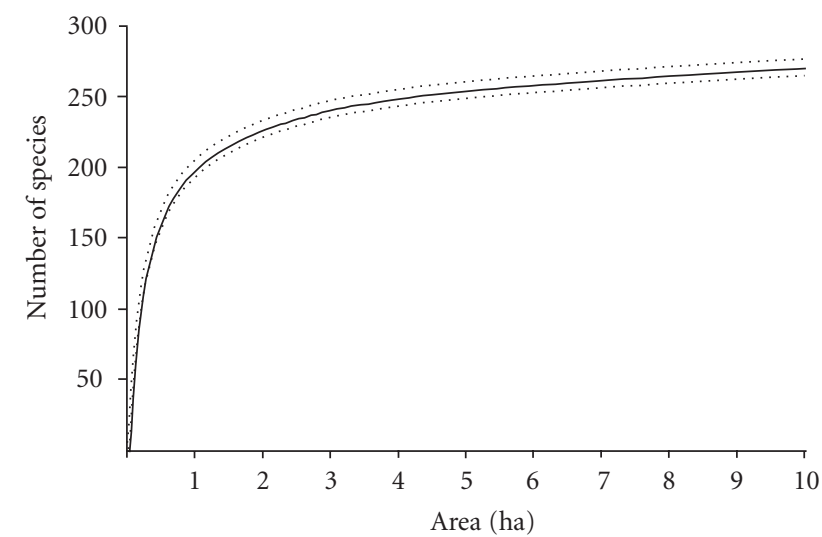

FIGURE 2: Sampling sufficiency designed by "jackknife" estimator for the ten sites of seasonal semideciduous forest in southeastern Brazil. Dashed line: standard deviation.

represented by only one genus. The best represented genera in terms of species number were Aspidosperma, Machaerium, and Ficus, with seven species each, followed by Ocotea, Cordia, Inga, Trichilia, and Casearia with four species each.

The total species richness, designed by jackknife estimators 1 and 2 and Chao 1 and 2 estimators, showed a similar pattern $(273,284,285$, and 268 species, resp.). Results suggest high regional tree species richness. The 242 species found approximates of richness designed for estimators, demonstrating sampling sufficiency among the ten hectares studied (Figure 2).

The Shannon's diversity index $\left(H^{\prime}\right)$ for each site showed diversity values between 2.92 and 3.97, with the Pielou's evenness index $\left(J^{\prime}\right)$ between 0.73 and 0.87 (Table 2). However, when we considered the ten sites as a single sample, Shannon's diversity index $\left(H^{\prime}\right)$ was 4.62 , and Pielou's evenness index was 0.84 . This value can be attributed to the sampling covering quite heterogeneous sites, allowing differences in remnant structure and floristic composition and, consequently, increasing beta diversity.

Only Copaifera langsdorffii and Hymenaea courbaril were presented with the highest frequency in distribution, reaching $100 \%$ of occurrence at the sites (Table 3 ). However, 75 species occurred in at least five studied fragments, highlighting Cordiera sessilis, Apuleia leiocarpa, Casearia gossypiosperma, Cheiloclinium cognatum, Ixora brevifolia, Luehea grandiflora, Protium heptaphyllum, Sweetia fruticosa, and Terminalia glabrescens which occurred in nine sites, and can be indicated as characteristic SSF species (Table 3). On the other hand, $31.8 \%$ (77) of species occurred at only one site.

The floristic similarity analysis between sites showed a similar pattern in seven of the ten sites examined (Figure 3 ). Accordingly, there was the formation of a floristically cohesive group (Group 1), where sites 3, 6, 7, 8, 9, and 10 were presented with values exceeding 0.5 , indicating sites that were floristically similar. Also, a second group was formed with sites 1 and 4, whereas site 2 was floristically different from all the other sites (Figure 3 ).

Detrended correspondence analysis, DCA, presented eigenvalues of 0.61 and 0.25 in the two first ordination axes,

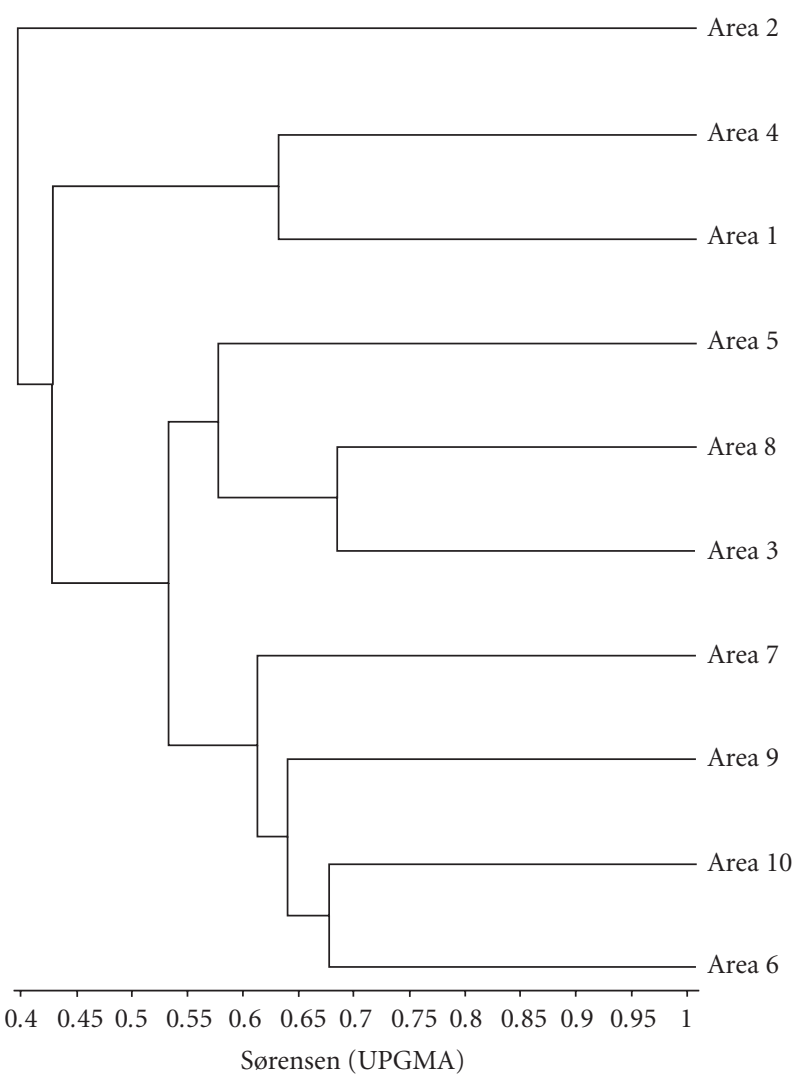

Figure 3: Dendrogram of similarity (Sørensen coefficient) produced by cluster analysis (UPGMA connection method) of tree species composition among the ten sites of seasonal semideciduous forest in southeastern Brazil.

explaining about $85 \%$ of the total data variation (Figure 4 ). The diagram demonstrated a large group formed from seven sites. Structural ordination, on the left, contemplates the same sites forming Group 1 by grouping analysis. These seven sites indicate the floristic pattern representative of the studied SSF. The second DCA axis was associated strongly with the sampled fragments' conservation degree. The group formed on the center left is characterized by intermediate conservation stages, while other sites on the top right are characterized by more advanced conservation stages (Group 2 ). On the lower right is the initial stage (site 2). Therefore, the three fragments on the right do not form a cohesive group. In addition, these three sites are those that are more distant from each other. Thus, the structural floristic similarity of the seven areas above may have been established on a geographical position basis. The division made by TWINSPAN corroborated the ordination results by DCA and the Sørensen coefficient (Figure 3).

The first division by TWINSPAN separated the seven SSF representative sites in this region from the three other fragments, owing to the absence of Siparuna guianensis, a species regarded as an indicator, which only occurs in the group formed on the other side of the dichotomy (Figure 5). In the second division, sites 3 and 8 were separated from the other fragments (Figure 5). The lower levels of division by TWINSPAN do not reveal new groups that make sense. 
TABLE 3: Species list sampled in ten sites of seasonal semideciduous forest of southeastern Brazil. NI = number of individuals; RF = frequency in the ten studied fragments; *First record for the Triangulo Mineiro region, ** First record for the Minas Gerais state in seasonal semideciduous forests, according [11].

\begin{tabular}{|c|c|c|}
\hline Families/Species & NI & $\mathrm{RF}(\%)$ \\
\hline \multicolumn{3}{|l|}{ Anacardiaceae } \\
\hline Astronium fraxinifolium Schott ex Spreng. & 18 & 40 \\
\hline Astronium nelson-rosae Santin & 257 & 60 \\
\hline Lithraea molleoides (Vell.) Engl. & 9 & 20 \\
\hline Myracrodruon urundeuva Allemão & 42 & 40 \\
\hline Tapirira guianensis Aubl. & 37 & 20 \\
\hline Tapirira obtusa (Benth.) J.D.Mitch. & 85 & 50 \\
\hline \multicolumn{3}{|l|}{ Annonaceae } \\
\hline Annona cacans Warm. & 29 & 50 \\
\hline Annona montana Macfad.* & 1 & 10 \\
\hline Cardiopetalum calophyllum Schltdl. & 6 & 40 \\
\hline Duguetia lanceolata A. St.-Hil. & 222 & 60 \\
\hline Guatteria australis A. St.-Hil.* & 28 & 10 \\
\hline Porcelia macrocarpa (Warm.) R. E. Fr.* & 1 & 10 \\
\hline Rollinia sylvatica (A. St.-Hil.) Mart. & 5 & 30 \\
\hline Unonopsis lindmanii R. E. Fr. & 102 & 40 \\
\hline Xylopia aromatica (Lam.) Mart. & 31 & 60 \\
\hline Xylopia brasiliensis Spreng. & 37 & 40 \\
\hline Xylopia sericea A. St.-Hil. & 1 & 10 \\
\hline \multicolumn{3}{|l|}{ Apocynaceae } \\
\hline Aspidosperma cuspa (Kunth) S. F. Blake ex Pittier & 10 & 10 \\
\hline Aspidosperma cylindrocarpon Müll. Arg. & 25 & 40 \\
\hline Aspidosperma discolor A. DC.* & 294 & 50 \\
\hline Aspidosperma olivaceum Müll. Arg. & 8 & 10 \\
\hline Aspidosperma parvifolium A. DC.* & 28 & 50 \\
\hline Aspidosperma polyneuron Müll. Arg. & 8 & 30 \\
\hline Aspidosperma subincanum Mart. ex A. DC. & 19 & 50 \\
\hline \multicolumn{3}{|l|}{ Aquifoliaceae } \\
\hline Ilex cerasifolia Reissek & 3 & 10 \\
\hline \multicolumn{3}{|l|}{ Araliaceae } \\
\hline Aralia warmingiana (Marchal) J. Wen & 12 & 30 \\
\hline Dendropanax cuneatus (DC.) Decne. \& Planch. & 14 & 20 \\
\hline Schefflera morototoni (Aubl.) Maguire et al. & 29 & 80 \\
\hline \multicolumn{3}{|l|}{ Arecaceae } \\
\hline Acrocomia aculeata (Jacq.) Lodd. ex Mart. & 5 & 10 \\
\hline \multicolumn{3}{|l|}{ Asteraceae } \\
\hline Piptocarpha macropoda Baker & 6 & 30 \\
\hline \multicolumn{3}{|l|}{ Bignoniaceae } \\
\hline Handroanthus serratifolia (Vahl) Nicholson & 22 & 70 \\
\hline Jacaranda cuspidifolia Mart. ex A. DC. & 2 & 20 \\
\hline Jacaranda macrantha Cham.* & 4 & 20 \\
\hline Tabebuia impetiginosus (Mart. ex DC.) Mattus & 2 & 20 \\
\hline Tabebuia roseoalba (Ridl.) Sandwith & 13 & 20 \\
\hline \multicolumn{3}{|l|}{ Boraginaceae } \\
\hline Cordia alliodora (Ruiz \& Pav.) Oken** & 1 & 10 \\
\hline Cordia sellowiana Cham. & 52 & 50 \\
\hline Cordia superba Cham. & 13 & 10 \\
\hline Cordia trichotoma (Vell.) Arrab. ex Steud. & 5 & 20 \\
\hline
\end{tabular}


Table 3: Continued.

\begin{tabular}{|c|c|c|}
\hline Families/Species & NI & $\mathrm{RF}(\%)$ \\
\hline \multicolumn{3}{|l|}{ Burseraceae } \\
\hline Protium heptaphyllum (Aubl.) Marchand & 282 & 90 \\
\hline \multicolumn{3}{|l|}{ Cannabaceae } \\
\hline Celtis iguanaea (Jacq.) Sarg. & 30 & 60 \\
\hline Trema micrantha (L.) Blume & 3 & 20 \\
\hline \multicolumn{3}{|l|}{ Cardiopteridaceae } \\
\hline Citronella paniculata (Mart.) R.A.Howard & 4 & 10 \\
\hline \multicolumn{3}{|l|}{ Caricaceae } \\
\hline Jacaratia spinosa (Aubl.) A. DC. & 4 & 20 \\
\hline \multicolumn{3}{|l|}{ Celastraceae } \\
\hline Cheiloclinium cognatum (Miers.) A. C. Sm. & 407 & 90 \\
\hline Maytenus floribunda Reissek* & 71 & 60 \\
\hline Maytenus robusta Reissek & 4 & 10 \\
\hline Maytenus sp. & 5 & 10 \\
\hline Salacia elliptica (Mart. ex Schult.) G. Don & 1 & 10 \\
\hline \multicolumn{3}{|l|}{ Chrysobalanaceae } \\
\hline Hirtella glandulosa Spreng. & 51 & 40 \\
\hline Hirtella gracilipes (Hook. f.) Prance & 72 & 50 \\
\hline Hirtella racemosa Lam. & 11 & 10 \\
\hline \multicolumn{3}{|l|}{ Clusiaceae } \\
\hline Calophyllum brasiliense Cambess. & 1 & 10 \\
\hline Garcinia brasiliensis Mart. & 64 & 60 \\
\hline \multicolumn{3}{|l|}{ Combretaceae } \\
\hline Terminalia argentea (Cambess.) Mart. & 2 & 10 \\
\hline Terminalia glabrescens Mart. & 202 & 90 \\
\hline Terminalia phaeocarpa Eichler & 56 & 60 \\
\hline \multicolumn{3}{|l|}{ Cunoniaceae } \\
\hline Lamanonia ternata Vell. & 12 & 20 \\
\hline \multicolumn{3}{|l|}{ Ebenaceae } \\
\hline Diospyros hispida A. DC. & 108 & 50 \\
\hline \multicolumn{3}{|l|}{ Elaeocarpaceae } \\
\hline Sloanea monosperma Vell. & 19 & 40 \\
\hline \multicolumn{3}{|l|}{ Erythroxylaceae } \\
\hline Erythroxylum daphnites Mart. & 6 & 10 \\
\hline Erythroxylum deciduum A. St.-Hil. & 3 & 10 \\
\hline \multicolumn{3}{|l|}{ Euphorbiaceae } \\
\hline Acalypha gracilis (Spreng.) Müll. Arg.** & 7 & 20 \\
\hline Alchornea glandulosa Poepp. \& Endl. & 20 & 30 \\
\hline Mabea fistulifera Mart. & 25 & 10 \\
\hline Maprounea guianensis Aubl. & 32 & 40 \\
\hline Micrandra elata Müll. Arg. & 118 & 10 \\
\hline Pera glabrata (Schott) Poepp. ex Baill. & 12 & 20 \\
\hline Sapium glandulosum (L.) Morong & 5 & 30 \\
\hline \multicolumn{3}{|l|}{ Fabaceae caesalpinoideae } \\
\hline Apuleia leiocarpa (Vogel) J. F. Macbr. & 114 & 90 \\
\hline Cassia ferruginea (Schrad.) Schrad. ex DC. & 9 & 30 \\
\hline Copaifera langsdorffii Desf. & 155 & 100 \\
\hline Hymenaea courbaril L. & 119 & 100 \\
\hline Peltophorum dubium (Spreng.) Taub.* & 2 & 20 \\
\hline Sclerolobium paniculatum Benth. & 3 & 10 \\
\hline
\end{tabular}


Table 3: Continued.

\begin{tabular}{|c|c|c|}
\hline Families/Species & $\mathrm{NI}$ & $\mathrm{RF}(\%)$ \\
\hline \multicolumn{3}{|l|}{ Fabaceae cercideae } \\
\hline Bauhinia rufa (Bong.) Steud. & 9 & 40 \\
\hline Bauhinia ungulata L.* & 20 & 50 \\
\hline \multicolumn{3}{|l|}{ Fabaceae faboideae } \\
\hline Andira fraxinifolia Benth. & 7 & 20 \\
\hline Andira ormosioides Benth.* & 1 & 10 \\
\hline Centrolobium tomentosum Guillem. ex Benth. & 1 & 10 \\
\hline Dipteryx alata Vogel & 5 & 20 \\
\hline Lonchocarpus cultratus (Vell.) Az.-Tozzi \& H. C. Lima & 11 & 30 \\
\hline Machaerium acutifolium Vogel & 7 & 30 \\
\hline Machaerium brasiliense Vogel & 69 & 70 \\
\hline Machaerium hirtum (Vell.) Stellfeld & 25 & 30 \\
\hline Machaerium nyctitans (Vell.) Benth. & 2 & 10 \\
\hline Machaerium opacum Vogel & 1 & 10 \\
\hline Machaerium stipitatum (DC.) Vogel & 20 & 60 \\
\hline Machaerium villosum Vogel & 62 & 60 \\
\hline Myroxylon peruiferum L. f. & 1 & 10 \\
\hline Ormosia arborea (Vell.) Harms & 23 & 70 \\
\hline Platycyamus regnellii Benth. & 50 & 30 \\
\hline Platypodium elegans Vogel & 32 & 70 \\
\hline Pterodon emarginatus Vogel & 2 & 10 \\
\hline Sweetia fruticosa Spreng. & 69 & 90 \\
\hline Vatairea macrocarpa (Benth.) Ducke & 1 & 10 \\
\hline Zollernia ilicifolia (Brongn.) Vogel & 21 & 40 \\
\hline \multicolumn{3}{|l|}{ Fabaceae Mimosoideae } \\
\hline Acacia polyphylla DC. & 37 & 70 \\
\hline Albizia niopoides (Spruce ex Benth.) Burkart & 23 & 30 \\
\hline Albizia polycephala (Benth.) Killip ex Record & 5 & 10 \\
\hline Anadenanthera colubrina (Vell.) Brenan & 67 & 20 \\
\hline Calliandra foliolosa Benth. & 5 & 10 \\
\hline Enterolobium contortisiliquum (Vell.) Morong & 9 & 40 \\
\hline Inga laurina (Sw.) Willd. & 9 & 50 \\
\hline Inga marginata Willd. & 10 & 10 \\
\hline Inga sessilis (Vell.) Mart.* & 44 & 60 \\
\hline Inga vera Willd. & 84 & 40 \\
\hline Piptadenia gonoacantha (Mart.) J. F. Macbr. & 167 & 50 \\
\hline \multicolumn{3}{|l|}{ Lacistemataceae } \\
\hline Lacistema aggregatum (P. J. Bergius) Rusby** & 3 & 20 \\
\hline \multicolumn{3}{|l|}{ Lamiaceae } \\
\hline Aegiphila sellowiana Cham. & 11 & 50 \\
\hline Vitex polygama Cham. & 6 & 20 \\
\hline \multicolumn{3}{|l|}{ Lauraceae } \\
\hline Cryptocarya aschersoniana $\mathrm{Mez}$ & 212 & 70 \\
\hline Endlicheria paniculata (Spreng.) J. F. Macbr. & 3 & 10 \\
\hline Nectandra cissiflora Nees & 44 & 30 \\
\hline Nectandra megapotamica (Spreng.) Mez & 29 & 50 \\
\hline Nectandra membranacea (Sw.) Griseb.* & 81 & 40 \\
\hline Ocotea corymbosa (Meisn.) Mez & 136 & 70 \\
\hline Ocotea minarum (Nees) Mez & 4 & 10 \\
\hline Ocotea pulchella Mart. & 8 & 10 \\
\hline Ocotea spixiana (Nees) Mez & 51 & 40 \\
\hline
\end{tabular}


TABle 3: Continued.

\begin{tabular}{|c|c|c|}
\hline Families/Species & NI & RF $(\%)$ \\
\hline \multicolumn{3}{|l|}{ Lecytidaceae } \\
\hline Cariniana estrellensis (Raddi) Kuntze & 51 & 70 \\
\hline \multicolumn{3}{|l|}{ Lythraceae } \\
\hline Lafoensia densiflora Pohl & 1 & 10 \\
\hline \multicolumn{3}{|l|}{ Malpiguiaceae } \\
\hline Byrsonima laxiflora Griseb. & 12 & 40 \\
\hline \multicolumn{3}{|l|}{ Malvaceae } \\
\hline Apeiba tibourbou Aubl. & 4 & 30 \\
\hline Ceiba speciosa (A. St.-Hil.) Ravenna & 19 & 40 \\
\hline Eriotheca candolleana (K. Schum.) A. Robyns & 28 & 50 \\
\hline Guazuma ulmifolia Lam. & 49 & 50 \\
\hline Luehea divaricata Mart. & 6 & 20 \\
\hline Luehea grandiflora Mart. \& Zucc. & 199 & 90 \\
\hline Pseudobombax tomentosum (Mart. \& Zucc.) A. Robyns & 3 & 20 \\
\hline Quararibea turbinata (Sw.) Poir.* & 5 & 10 \\
\hline \multicolumn{3}{|l|}{ Melastomataceae } \\
\hline Miconia cuspidata Mart. ex Naudin & 1 & 10 \\
\hline Miconia latecrenata (DC.) Naudin & 10 & 30 \\
\hline Miconia minutiflora (Bonpl.) DC.* & 7 & 10 \\
\hline \multicolumn{3}{|l|}{ Meliaceae } \\
\hline Cabralea canjerana (Vell.) Mart. & 7 & 40 \\
\hline Cedrela fissilis Vell. & 16 & 40 \\
\hline Guarea guidonia (L.) Sleumer & 23 & 50 \\
\hline Guarea kunthiana A. Juss. & 19 & 20 \\
\hline Trichilia catigua A. Juss. & 165 & 80 \\
\hline Trichilia claussenii C. DC. & 130 & 20 \\
\hline Trichilia elegans A. Juss. & 55 & 70 \\
\hline Trichilia pallida Sw. & 28 & 70 \\
\hline \multicolumn{3}{|l|}{ Monimiaceae } \\
\hline Mollinedia widgrenii A. DC. & 6 & 30 \\
\hline \multicolumn{3}{|l|}{ Moraceae } \\
\hline Ficus clusiifolia Schott* & 2 & 10 \\
\hline Ficus guaranitica Chodat & 6 & 40 \\
\hline Ficus obtusiuscula (Miq.) Miq. & 1 & 10 \\
\hline Ficus pertusa L. f.* & 1 & 10 \\
\hline Ficus trigona L. f. & 3 & 20 \\
\hline Ficus sp1 & 1 & 10 \\
\hline Ficus sp 2 & 1 & 10 \\
\hline Maclura tinctoria (L.) Steud. & 4 & 30 \\
\hline Pseudolmedia laevigata Trécul & 5 & 20 \\
\hline Sorocea bonplandii (Baill.) W. C. Burger et al. & 9 & 40 \\
\hline \multicolumn{3}{|l|}{ Myristicaceae } \\
\hline Virola sebifera Aubl. & 127 & 80 \\
\hline \multicolumn{3}{|l|}{ Myrtaceae } \\
\hline Calyptranthes clusiifolia O. Berg & 5 & 30 \\
\hline Calyptranthes widgreniana O. Berg & 7 & 20 \\
\hline Campomanesia guazumifolia (Cambess.) O. Berg & 1 & 10 \\
\hline Campomanesia velutina (Cambess.) O. Berg & 93 & 70 \\
\hline Eugenia florida DC. & 190 & 50 \\
\hline Eugenia involucrata DC. & 42 & 40 \\
\hline
\end{tabular}


TABle 3: Continued.

\begin{tabular}{|c|c|c|}
\hline Families/Species & NI & $\mathrm{RF}(\%)$ \\
\hline Eugenia ligustrina (Sw.) Willd. & 27 & 30 \\
\hline Eugenia subterminalis DC.* & 20 & 20 \\
\hline Gomidesia lindeniana O. Berg & 1 & 10 \\
\hline Myrcia splendens (Sw.) DC. & 11 & 60 \\
\hline Myrcia tomentosa (Aubl.) DC. & 9 & 40 \\
\hline Myrciaria glanduliflora (Kiaersk.) Mattos \& D. Legrand** & 106 & 40 \\
\hline Myrciaria tenella (DC.) O. Berg & 2 & 10 \\
\hline Psidium longipetiolatum D. Legrand** & 2 & 10 \\
\hline Psidium rufum DC. & 12 & 40 \\
\hline Psidium sartorianum (O. Berg) Nied. & 41 & 50 \\
\hline Siphoneugena densiflora O. Berg & 133 & 60 \\
\hline Syzygium jambos (L.) Aston.* & 1 & 10 \\
\hline Myrtaceae 1 & 1 & 10 \\
\hline Myrtaceae 2 & 1 & 10 \\
\hline \multicolumn{3}{|l|}{ Nyctaginaceae } \\
\hline Guapira opposita (Vell.) Reitz & 28 & 10 \\
\hline Guapira venosa (Choisy) Lundell & 54 & 40 \\
\hline Neea hermaphrodita S. Moore** & 12 & 10 \\
\hline \multicolumn{3}{|l|}{ Ochnaceae } \\
\hline Ouratea castaneifolia (DC.) Engl. & 12 & 50 \\
\hline \multicolumn{3}{|l|}{ Olacaceae } \\
\hline Heisteria ovata Benth. & 113 & 60 \\
\hline \multicolumn{3}{|l|}{ Oleaceae } \\
\hline Chionanthus trichotomus (Vell.) P. S. Green & 3 & 10 \\
\hline \multicolumn{3}{|l|}{ Opiliaceae } \\
\hline Agonandra brasiliensis Miers ex Benth. \& Hook. & 24 & 50 \\
\hline \multicolumn{3}{|l|}{ Phyllanthaceae } \\
\hline Margaritaria nobilis L. f. & 27 & 70 \\
\hline Phyllanthus acuminatus Vahl & 2 & 10 \\
\hline \multicolumn{3}{|l|}{ Piperaceae } \\
\hline Piper amalago L. & 4 & 10 \\
\hline Piper arboreum Aubl. & 2 & 10 \\
\hline \multicolumn{3}{|l|}{ Polygonaceae } \\
\hline Coccoloba mollis Casar. & 9 & 40 \\
\hline \multicolumn{3}{|l|}{ Primulaceae } \\
\hline Ardisia ambigua Mez & 25 & 30 \\
\hline Myrsine coriacea (Sw.) Roem. \& Schult. & 2 & 10 \\
\hline Myrsine leuconeura Mart. & 2 & 20 \\
\hline Myrsine umbellata Mart. & 15 & 20 \\
\hline \multicolumn{3}{|l|}{ Proteaceae } \\
\hline Roupala brasiliensis Klotzsch & 31 & 80 \\
\hline \multicolumn{3}{|l|}{ Rhaminaceae } \\
\hline Rhamnidium elaeocarpum Reissek & 34 & 50 \\
\hline \multicolumn{3}{|l|}{ Rubiaceae } \\
\hline Amaioua guianensis Aubl. & 35 & 40 \\
\hline Chomelia pohliana Mull. Arg. & 14 & 40 \\
\hline Cordiera sessilis (Vell.) Kuntze & 404 & 90 \\
\hline Coussarea hydrangeifolia (Benth.) Müll. Arg. & 32 & 60 \\
\hline Coutarea hexandra (Jacq.) K. Schum. & 21 & 70 \\
\hline Faramea hyacinthina Mart. & 43 & 50 \\
\hline Genipa americana L. & 1 & 10 \\
\hline
\end{tabular}


Table 3: Continued.

\begin{tabular}{|c|c|c|}
\hline Families/Species & NI & $\mathrm{RF}(\%)$ \\
\hline Guettarda viburnoides Cham. \& Schltdl. & 29 & 50 \\
\hline Ixora brevifolia Benth. & 131 & 90 \\
\hline Machaonia brasiliensis (Hoffmanss. ex Humb.) Cham. \& Schltdl.* & 2 & 10 \\
\hline Rudgea viburnoides (Cham.) Benth. & 11 & 50 \\
\hline Simira sampaioana (Standl.) Steyerm.* & 47 & 70 \\
\hline Tocoyena formosa (Cham. \& Schltdl.) K.Schum. & 1 & 10 \\
\hline \multicolumn{3}{|l|}{ Rutaceae } \\
\hline Galipea jasminiflora (A. St.-Hil.) Engl.* & 142 & 10 \\
\hline Metrodorea nigra A. St.-Hil. & 10 & 20 \\
\hline Metrodorea stipularis Mart. & 6 & 10 \\
\hline Pilocarpus spicatus A. St.-Hil.* & 1 & 10 \\
\hline Zanthoxylum riedelianum Engl. & 11 & 30 \\
\hline \multicolumn{3}{|l|}{ Salicaceae } \\
\hline Casearia gossypiosperma Briq. & 172 & 90 \\
\hline Casearia grandiflora Cambess. & 218 & 40 \\
\hline Casearia rupestris Eichler & 8 & 10 \\
\hline Casearia sylvestris Sw. & 65 & 60 \\
\hline Prockia crucis P. Browne ex L. & 5 & 20 \\
\hline Xylosma prockia (Turcz.) Turcz.* & 1 & 10 \\
\hline \multicolumn{3}{|l|}{ Sapindaceae } \\
\hline Allophylus edulis (A. St.-Hil., Cambess. \& A.Juss.) Radlk. & 1 & 10 \\
\hline Allophylus racemosus Sw. & 8 & 30 \\
\hline Cupania vernalis Cambess. & 78 & 80 \\
\hline Dilodendron bipinnatum Radlk. & 10 & 20 \\
\hline Magonia pubescens A. St.-Hil. & 2 & 10 \\
\hline Matayba elaeagnoides Radlk.** & 39 & 40 \\
\hline Matayba guianensis Aubl. & 105 & 70 \\
\hline \multicolumn{3}{|l|}{ Sapotaceae } \\
\hline Chrysophyllum gonocarpum (Mart. \& Eichler) Engl. & 69 & 40 \\
\hline Chrysophyllum marginatum (Hook. \& Arn.) Radlk. & 161 & 20 \\
\hline Micropholis venulosa (Mart. \& Eichler) Pierre & 61 & 30 \\
\hline Pouteria gardneri (Mart. \& Miq.) Baehni & 42 & 70 \\
\hline Pouteria torta (Mart.) Radlk. & 128 & 80 \\
\hline \multicolumn{3}{|l|}{ Siparunaceae } \\
\hline Siparuna guianensis Aubl. & 407 & 70 \\
\hline \multicolumn{3}{|l|}{ Styracaceae } \\
\hline Styrax camporum Pohl & 35 & 50 \\
\hline \multicolumn{3}{|l|}{ Symplocaceae } \\
\hline Symplocos pubescens Klotzsch ex Benth.* & 8 & 10 \\
\hline \multicolumn{3}{|l|}{ Urticaceae } \\
\hline Cecropia pachystachya Trécul & 11 & 30 \\
\hline Urera baccifera (L.) Gaudich. ex Wedd. & 10 & 10 \\
\hline \multicolumn{3}{|l|}{ Verbenaceae } \\
\hline Aloysia virgata (Ruiz \& Pav.) A. Juss. & 3 & 20 \\
\hline \multicolumn{3}{|l|}{ Vochysiacaeae } \\
\hline Callisthene major Mart. & 62 & 40 \\
\hline Qualea dichotoma (Mart.) Warm. & 5 & 30 \\
\hline Qualea jundiahy Warm.* & 39 & 60 \\
\hline Vochysia magnifica Warm. & 114 & 30 \\
\hline Vochysia tucanorum Mart. & 5 & 10 \\
\hline
\end{tabular}




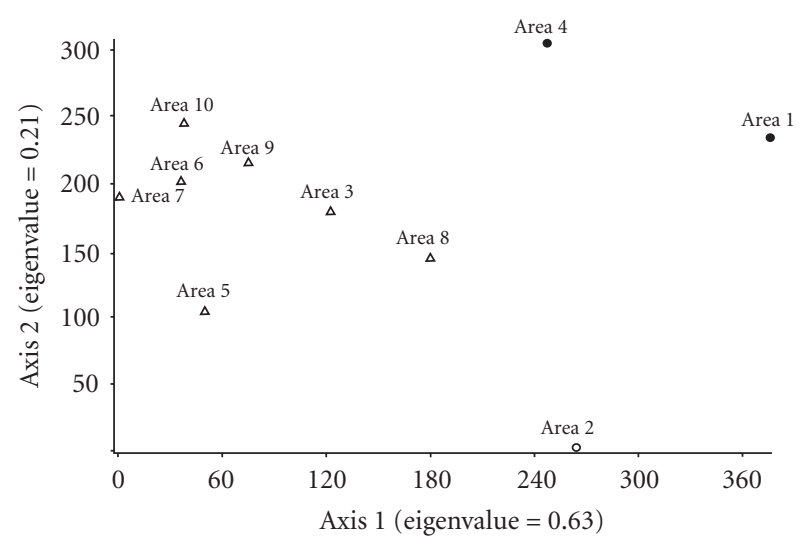

Figure 4: Ordination diagram in first two axes of detrended correspondence analysis (DCA) of ten sites of seasonal semideciduous forest in southeastern Brazil. Symbols correspond to floristic groups $1(\triangle), 2(\bullet)$, and $3(\mathrm{o})$.

\section{Discussion}

Studies conducted in areas of SSF in Brazil presented Fabaceae, Myrtaceae, Rubiaceae, Annonaceae, Lauraceae, and Meliaceae as the families with greater species richness [33-36]. In São Paulo state SSF, as well as the greater richness of families found in this study, there is also an emphasis on Melastomataceae and Solanaceae [37, 38]. In northeastern Brazil, the most prominent families in terms of species number in SSF sites are Fabaceae Mimosoideae, Euphorbiaceae, Fabaceae Faboideae, Myrtaceae, and Rubiaceae [39]. The pattern in families and genera richness found here corroborates that described by Oliveira Filho and Fontes [40] for SSF domains in southeastern Brazil, with significant tree species richness in Fabaceae (all subfamilies), Lauraceae, Myrtaceae, Moraceae, and Rubiaceae and genres Aspidosperma, Ficus, Machaerium, and Ocotea.

Copaifera langsdorffii and $H$. courbaril are wide-ranging adaptive species, occurring in various forest formations of the Cerrado region, as well as in other geographical provinces in Brazil and are, therefore, considered to be habitat generalists [41]. Apuleia leiocarpa and Luehea grandiflora are also among the most frequent species of SSF, according to a study presented by Ferreira Júnior et al. [42], which brought together 15 studies in southeastern Brazil. The tree species composition studied in SSF showed a relationship with lowland seasonal forests of Brazil's eastern Minas Gerais state, based on the indicator species analysis made by Oliveira-Filho and Fontes [40]. Of the 57 species identified by these authors as eastern Minas Gerais region indicators, $36(63.1 \%)$ were recorded in the sample sites presented here. This data corroborates the idea that interior SSF fragments may have a floristic connection with the Atlantic forest that is able to expand its distribution in sites with strong seasonality by means of gallery forests [43].

The presence of unique species to each location highlights the heterogeneity between samples (sites), reflected in increased beta diversity. We should point out that the limited number of individuals in some populations may impair the biological conservation of many species, causing serious difficulties for their preservation. However, some species, such as Galipea jasminiflora and Micrandra elata, occurred in only one fragment, but with a high density. This observation acquires relevance in terms of plant population conservation. The conservation of the remaining forest, with different structures and consequent higher beta diversity, can be a relevant parameter to the decision for choosing priority areas for conservation in SSF [44].

Diversity values are among those frequently found for SSF and Atlantic rainforest, which generally vary between 3.2 and 4.2 [45]. However, they are lower than those found in other tropical forests [46]. The lower values for evenness found in fragments indicate a relatively high concentration in density of a small number of species, which dominate the tree community. The predominance of a few species, in number or biomass in a community (also known as ecological dominance), is not uncommon in tropical forests [47].

A variation in similarity values occurs as a result of different conditions in the use and historic occupation of each fragment and acts as an important force capable of modifying plant communities through spatial and temporal heterogeneity, thus determining the community composition and structure $[16,48]$. The separation of site 2 from other areas, along with Group 2, can be related to different stages of forest succession. Site 2 presents in an initial successional stage owing to an intense human processes. This fragment, although situated in the countryside, reveals recent historical disturbance as a result of selective logging and the presence of cattle, and the surrounding matrix is formed entirely by agricultural sites [49]. Selective logging enables the creation of gaps within the fragment and the establishment of pioneering species. As Whitmore states $[47,50]$, pioneer trees and shrubs need high light and temperature intensities for seed germination and seedling establishment and growth. Group 2 (sites 1 and 4) is formed by the more well-preserved fragments among those studied and is characterized by the presence and dominance of individuals with a large basal area. In fragment 4, for example, Micrandra elata was sampled with a density of 118 ind $\cdot \mathrm{ha}^{-1}$ and a basal area of $24.51 \mathrm{~m}^{2} \cdot \mathrm{ha}^{-1}$ [51], whereas in fragment 1, Psidium sartorianum was the species with the highest dominance [52].

Although the floristic similarity between the fragments can be related to the geographical proximity, as already reported by MaCdonald et al. [53], we verified that similarity relations between studied sites are established mainly as a result of the community successional stage. This same pattern was also found by Durigan et al. [17]; that is, within the same vegetal formation, communities at similar stages of the successional process tend to have similar flora.

This analysis showed high beta diversity of the studied SSF arboreal flora, mainly as a result of the coverage of heterogeneous areas in relation to fragments at a successional stage. The largest SSF sampling in the Cerrado domain led to the registration and distribution of several plant populations, which had previously only been described for the Atlantic forests domain, demonstrating not only a lack of studies 


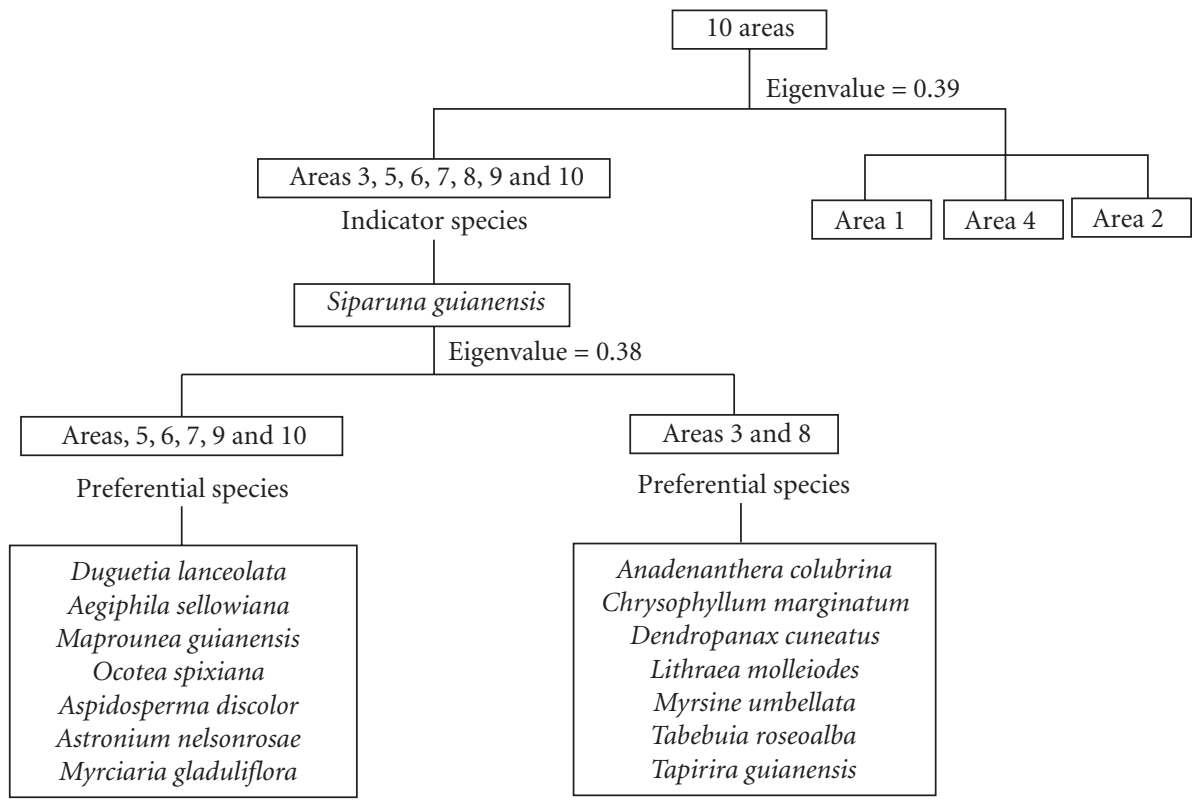

FIgURE 5: Classification by TWINSPAN of ten sites of seasonal semideciduous forest in southeastern Brazil.

like this, but also corroborating the idea of strong floristic connections with rainforests in the east of the country [40].

The fragmentation process started in the 1970s in the studied region of Brazil, especially as a result of the incentive given to landowners by the public authority for the advancement of occupied areas for agricultural and livestock activities, which had a direct consequence for the formation of many smaller forest fragments. Fragmentation possibly created similar processes in forests' successional development, which has conditioned a floristic pattern as found for seven of the ten sites surveyed.

\section{Acknowledgments}

Thanks are due to all those who assisted fieldwork and Professor Dr. Glein Monteiro de Araújo for aid in the field and in species identification. The authors would like to thank Fundo de Amparo a Pesquisa do Estado de Minas Gerais (FAPEMIG) for financial support for this Project (Process no. APQ-00694-08).

\section{References}

[1] L. O. Silva, D. A. Costa, K. E. Santo-Filho, H. D. Ferreira, and D. Brandão, "Levantamento florístico e fitossociológico em duas áreas de cerrado sensu stricto no Parque Estadual da Serra de Caldas Novas, Goiás," Acta Botanica. Brasilica, vol. 16, no. 2, pp. 43-53, 2002.

[2] L. Miles, A. C. Newton, R. S. DeFries et al., "A global overview of the conservation status of tropical dry forests," Journal of Biogeography, vol. 33, no. 3, pp. 491-505, 2006.

[3] D. E. Prado and P. E. Gibbs, "Patterns of species distribution in the dry seasonal forests of South America," Annual Missouri Botanical Garden, vol. 80, no. 4, pp. 902-927, 1993.
[4] R. T. Pennington, D. E. Prado, and C. A. Pendry, "Neotropical seasonally dry forests and Quaternary vegetation changes," Journal of Biogeography, vol. 27, no. 2, pp. 261-273, 2000.

[5] D. E. Prado, "Seasonally dry forests of tropical South America: From forgotten ecosystems to a new phytogeographic unit," Edinburgh Journal of Botany, vol. 57, no. 3, pp. 437-461, 2000.

[6] M. J. N. Rodal, "Montane forests in Northeast Brazil: a phytogeographical approach," Botanishe Jahrbücher für Systematik, Pflanzengeschichte und Pflanzengeographie, vol. 124, no. 1, pp. $1-10,2002$.

[7] M. J. N. Rodal and L. M. Nascimento, "Levantamento florístico da floresta serrana da Reserva Biológica de Serra Negra, microrregião de Itaparica, Pernambuco, Brasil," Acta Botanica Brasilica, vol. 16, no. 4, pp. 481-500, 2002.

[8] R. Spichiger, R. Palese, A. Chautems, and L. Ramella, "Origin, affinities and diversity hot spots of the Paraguayan dendrofloras," Candollea, vol. 50, no. 2, pp. 515-537, 1995.

[9] F. L. F. Quadros and V. P. Pillar, "Transições floresta-campo no Rio Grande do Sul: Hipóteses sobre clima e interação fogovegetação," Ciência \& Ambiente, vol. 24, no. 5, pp. 109-118, 2002.

[10] J. F. Silva, M. R. Fariñas, J. M. Felfili, and C. A. Klink, "Spatial heterogeneity, land use and conservation in the cerrado region of Brazil," Journal of Biogeography, vol. 33, no. 3, pp. 536-548, 2006.

[11] A. T. Oliveira Filho, Catálogo das árvores nativas de Minas Gerais: mapeamento e inventário da flora nativa e dos reflorestamentos de Minas Gerais, Editora UFLA, Lavras, Brazil, 2006.

[12] J. R. Scolforo and L. M. T. Carvalho, Mapeamento e inventário da flora nativa e dos reflorestamentos de Minas Gerais, UFLA, Lavras, Brasil, 2006.

[13] J. M. Felfili, P. E. Nogueira, M. C. Silva Júnior, B. S. Marimon, and W. B. C. Delitti, "Composição florística e fitossociologia do cerrado sentido restrito no município de Água Boa, MT," Acta Botanica Brasilica, vol. 16, no. 1, pp. 103-112, 2002.

[14] F. M. V. Carvalho, P. De Marco, and L. G. Ferreira, "The Cerrado into-pieces: habitat fragmentation as a function of 
landscape use in the savannas of central Brazil," Biological Conservation, vol. 142, no. 7, pp. 1392-1403, 2009.

[15] K. A. Ross, B. J. Fox, and M. D. Fox, "Changes to plant species richness in forest fragments: fragment age, disturbance and fire history may be as important as area," Journal of Biogeography, vol. 29, no. 5-6, pp. 749-765, 2002.

[16] T. C. Whitmore and D. F. R. P. Burslem, "Major disturbances in tropical rainforests," in Dynamics of Tropical Communities, D. M. Newbery, H. H. T. Prins, and N. Brown, Eds., Blackwell, Oxford, UK, 1998.

[17] G. Durigan, L. C. Bernacci, G. A. D. C. Franco, G. D. F. Arbocz, J. P. Metzger, and E. L. M. Catharino, "Successional stage and geographic features determine floristic similarity among Atlantic Forest remnants, São Paulo State, BrazilEstádio sucessional e fatores geográficos como determinantes da similaridade florística entre comunidades florestais no Planalto Atlântico, Estado de São Paulo, Brasil," Acta Botanica Brasilica, vol. 22, no. 1, pp. 51-62, 2008.

[18] H. P. Veloso, A. L. R. Rangel Filho, and J. C. A. Lima, Classificação da Vegetação Brasileira Adaptada a um Sistema Universal, IBGE, Rio de Janeiro, Brasil, 1991.

[19] A. N. Ab’saber, "Contribuição a Geomorfologia da área dos Cerrados," in Simpósio sobre o Cerrado, EDUSP, São Paulo, Brazil, 1971.

[20] Radam Brasil, Levantamentos de Recursos Naturais, Folha, Rio de Janeiro, Brazil, 1983.

[21] EMBRAPA - Empresa Brasileira de Pesquisa Agropecuária, Sistema brasileiro de classificação de solos, EMBRAPA Produção de Informação, Brasília, Brazil, 1999.

[22] W. Koeppen, Climatología, Editora Fondo de La Cultura Económica, México, 1948.

[23] K. A. Alves and R. Rosa, "Espacialização de dados climáticos do cerrado mineiro," 2008, http://www.horizontecientifico .propp.ufu.br/.

[24] Missouri Botanical Garden, "MOBOT," 2010, http://www. mobot.org/W3T/search/vasthtml/.

[25] APG III, "An update of the Angiosperm Phylogeny Group classification for the orders and families of flowering plants: APG III," Botanical Journal of the Linnean Society, vol. 161, pp. 105-121, 2009.

[26] J. F. Heltshe and N. E. Forrester, "Estimating species richness using the jackknife procedure," Biometrics, vol. 39, no. 1, pp. $1-11,1983$.

[27] R. K. Collwell, "EstimateS: estatistical estimation of species richness and shared species from samples. Version 8.0," 2006, http://viceroy.eeb.uconn.edu/EstimateS.

[28] G. J. Shepherd, FITOPAC 1.6, Departamento de Botânica, UNICAMP, 2006.

[29] J. E. Brower and J. H. Zar, Field \& Laboratory Methods for General Ecology, Brown Publishers, Boston, Mass, USA, 1984.

[30] M. O. Hill and H. G. Gauch Jr., "Detrended correspondence analysis: an improved ordination technique," Vegetatio, vol. 42, no. $1-3$, pp. $47-58,1980$.

[31] M. O. Hill, TWINSPAN. A Fortran Program for Arranging Multivariate Data in an Ordered Two-Way Table by Classification of the Individuals and Attributes, Cornell University, Ithaca, NY, USA, 1979.

[32] B. MacCune and M. J. Mefford, PC-ORD. Multivariate Analysis of Ecological Data, Version 4, MjM Software Design, Gleneden Beach, Ore, USA, 1999.

[33] A. T. Oliveira-Filho, E. A. Vilela, M. L. Gavilanes, and D. A. Carvalho, "Comparison of the woody flora and soils of six areas of montane semideciduous Forest in Southern Minas Gerais, Brazil," Edinburgh Journal Botany, vol. 51, no. 3, pp. 355-389, 1994.
[34] E. A. Vilela, A.T. Oliveira Filho, D. A. Carvalho, and M. L. Gavilanes, "Flora arbustivo-arbóreo de um fragmento de mata ciliar no Alto Rio Grande, Itutinga, Minas Gerais," Acta Botanica Brasilica, vol. 9, no. 1, pp. 87-100, 1995.

[35] G. Pedralli, V. L. O. Freitas, S. T. Meyer, M. C. B. Teixeira, and A. P. S. Gonçalves, "Levantamento florístico na Estação Ecológica do Tripuí, Ouro Preto, MG," Acta Botanica Brasilica, vol. 11, no. 2, pp. 191-213, 1997.

[36] M. S. Werneck, G. Pedralli, R. Koenig, and L. F. Giseke, "Florística e estrutura de três trechos de uma floresta semidecídua na Estação Ecológica do Tripuí, Ouro Preto, MG," Revista Brasileira de Botânica, vol. 23, no. 1, pp. 97-106, 2000.

[37] S. N. Pagano and H. F. Leitão Filho, "Composição florística do estrato arbóreo de mata mesófila semidecídua, no município de Rio Claro (Estado de São Paulo)," Revista Brasileira de Botânica, vol. 10, no. 1, pp. 37-47, 1987.

[38] L. A. Silva and J. J. Soares, "Composição florística de um fragmento de floresta estacional semidecídua no município de São Carlos-SP," Revista Árvore, vol. 27, no. 5, pp. 647-656, 2003.

[39] M. J. M. Rodal, M. F. A. Lucena, K. V. S. A. Andrade, and A. L. Melo, "Mata do Toró: uma floresta estacional semidecidual de terras baixas no nordeste do Brasil," Hoehnea, vol. 32, no. 2, pp. 283-294, 2000.

[40] A. T. Oliveira-Filho and M. A. L. Fontes, "Patterns of floristic differentiation among atlantic forests in southeastern Brazil and the influence of climate," Biotropica, vol. 32, no. 4 B, pp. 793-810, 2001.

[41] A. T. Oliveira Filho and J. A. Ratter, "Padrões florísticos das matas ciliares da região do Cerrado e a evolução das paisagens do Brasil Central durante o Quaternário Tardio," in Matas Ciliares: Conservação e recuperação, R. R. Rodrigues and H. Leitão-Filho, Eds., EDUSP, São Paulo, Brazil, 2000.

[42] W. G. Ferreira Júnior, A. F. Silva, J. A. A. Meira Neto et al., "Composição florística da vegetação de um trecho de floresta estacional semidecídua em Viçosa, Minas Gerais, e espécies de maior ocorrência na região," Revista Árvore, vol. 31, no. 6, pp. 1121-1130, 2007.

[43] A. T. Oliveira-Filho and J. A. Ratter, "A study of the origin of central Brazilian forests by the analysis of plant species distribution patterns," Edinburgh Journal of Botany, vol. 52, no. 2, pp. 141-194, 1995.

[44] S. F. Lopes, V. S. Vale, A. P. Oliveira, and I. Schiavini, "Análise comparativa da estrutura e composição florística de cerrado no Brasil Central," Interciência, vol. 36, no. 1, pp. 8-15, 2011.

[45] J. A. A. Meira Neto and F. R. Martins, "Estrutura da mata da silvicultura, uma floresta estacional semidecidual montana no Município de Viçosa - MG," Revista Árvore, vol. 24, no. 2, pp. 151-160, 2000.

[46] D. H. Knight, "A Phytosociological analysis of species-rich tropical forest on Barro Colorado Island, Panamá," Ecological Monographs, vol. 45, no. 3, pp. 269-284, 1975.

[47] T. C. Whitmore, An Introduction to Tropical Rain Forests, Clarendon Press, Oxford, UK, 1990.

[48] E. Van der Maarel, "Some remarks on disturbance and its relation to diversity and stability," Journal Vegetation Science, vol. 4, pp. 733-736, 1993.

[49] A. E. Gusson, S. F. Lopes, O. C. Dias Neto, V. S. Vale, A. P. Oliveira, and I. Schiavini, "Características químicas do solo e estrutura de um fragmento de Floresta Estacional Semidecidual em Ipiaçu, Minas Gerais, Brasil," Rodriguésia, vol. 60, no. 2, pp. 403-414, 2009. 
[50] T. C. Whitmore, "A review of some aspects of tropical rain forest seedling ecology with suggestions for further enquiry," in The Ecology of Tropical Forest Seedlings, M. D. Swaine, Ed., UNESCO and Parthenon Publishing Group, Paris, France, 1996.

[51] O. C. Dias Neto, I. Schiavini, S. F. Lopes, V. S. Vale, A. E. Gusson, and A. P. Oliveira, "Estrutura fitossociológica e grupos ecológicos em um fragmento de floresta estacional semidecidual, Uberaba, Minas Gerais, Brasil," Rodriguésia, vol. 60, no. 4, pp. 1087-1100, 2009.

[52] V. S. Vale, I. Schiavini, S. F. Lopes, O. C. Dias Neto, A. P. Oliveira, and A. E. Gusson, "Floristic composition and structure of the tree component in a remnant of primary forest in semideciduous Araguari, Minas Gerais, Brazil," Hoehnea, vol. 36, no. 2, pp. 417-429, 2009.

[53] R. McDonald, M. McKnight, D. Weiss et al., "Species compositional similarity and ecoregions: do ecoregion boundaries represent zones of high species turnover?" Biological Conservation, vol. 126, no. 1, pp. 24-40, 2005. 

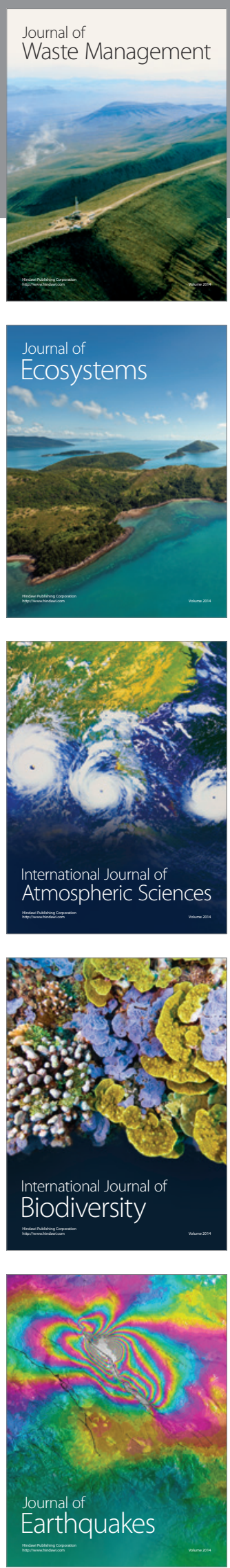
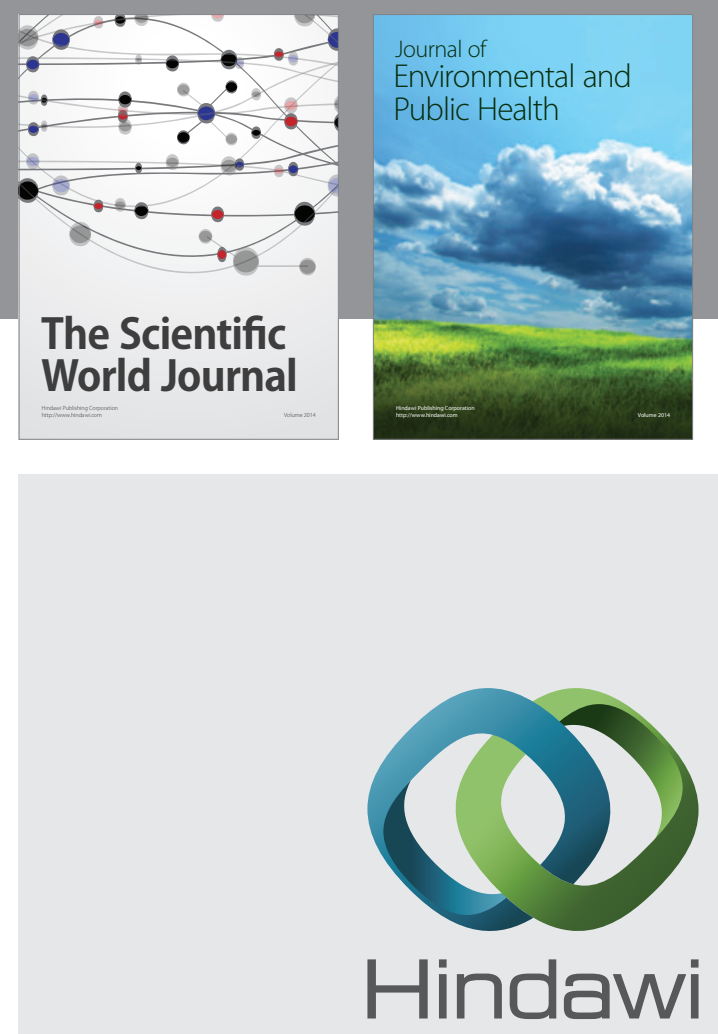

Submit your manuscripts at

http://www.hindawi.com
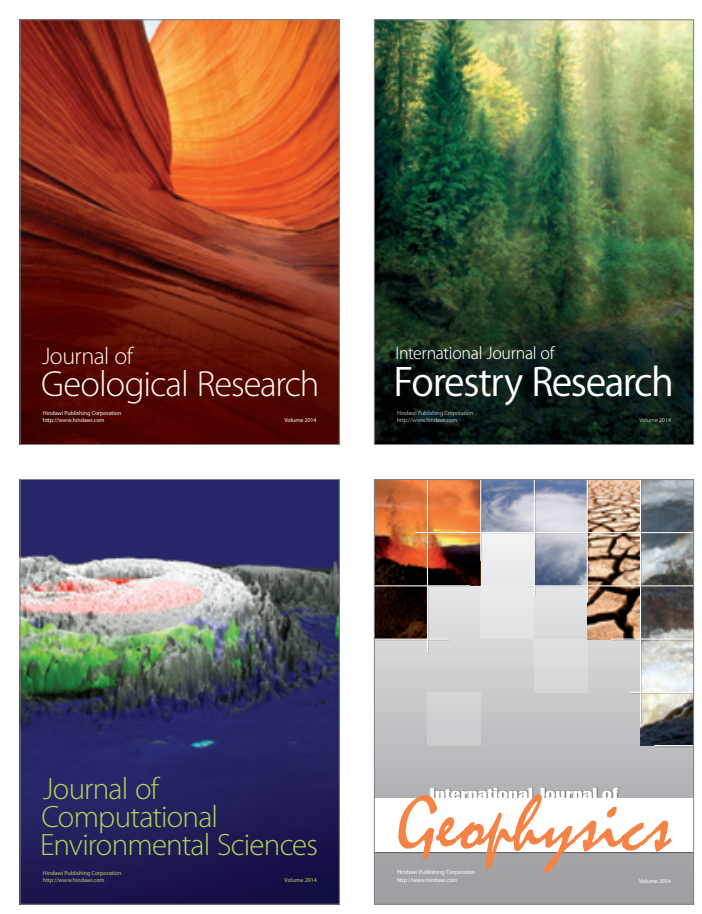
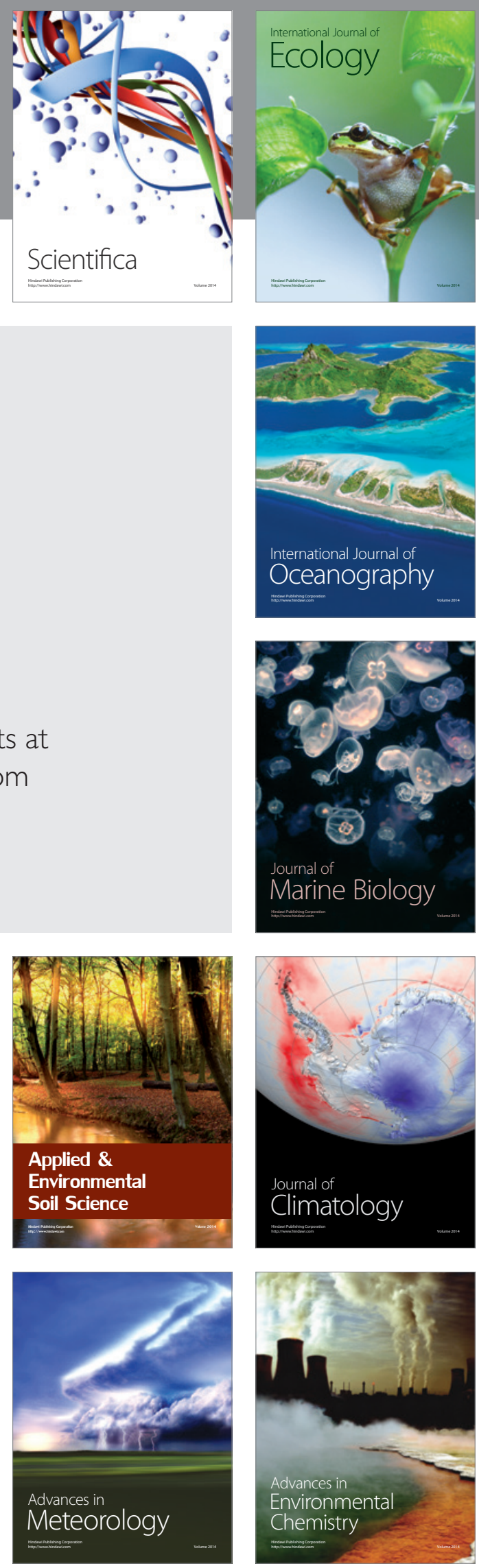